\title{
The metric growth of the discrete Laplacian
}

\author{
Hisayasu KURATA and Maretsugu YAMASAKI
}

(Received May 13, 2014; Revised December 24, 2014)

\begin{abstract}
Networks play important roles in the theory of discrete potentials. Especially, the theory of Dirichlet spaces on networks has become one of the most important tools for the study of potentials on networks. In this paper, first we study some relations between the Dirichlet sums of a function and of its Laplacian. We introduce some conditions to investigate properties of several functional spaces related to Dirichlet potentials and to biharmonic functions. Our goal is to study the growth of the Laplacian related to biharmonic functions on an infinite network. As an application, we prove a Riesz Decomposition theorem for Dirichlet functions satisfying various conditions.
\end{abstract}

Key words: discrete potential theory, discrete Laplacian, Riesz Decomposition.

\section{Introduction with preliminaries}

Let $N=\langle X, Y, K, r\rangle$ be an infinite network which is connected and locally finite and has no self-loop. Here $X$ is the set of nodes, $Y$ is the set of arcs, $K$ is a node-arc incidence matrix, and the resistance $r$ is a strictly positive function on $Y$. For $x \in X$ and for $y \in Y$ we let

$$
\begin{gathered}
e(y)=\{x \in X: K(x, y) \neq 0\}, \\
Y(x)=\{y \in Y: K(x, y) \neq 0\} .
\end{gathered}
$$

Note that $e(y)$ consists of exactly two nodes $x_{+}$and $x_{-}$, which satisfy $K\left(x_{+}, y\right)=1$ and $K\left(x_{-}, y\right)=-1$.

Let $L(X)$ and $L(Y)$ be the sets of all real-valued functions on $X$ and $Y$ respectively and let $L_{0}(X)$ be the set of all $u \in L(X)$ with finite support. For every $u, v \in L(X)$, let us put

$$
\begin{aligned}
d u(y) & =-r(y)^{-1} \sum_{x \in X} K(x, y) u(x) \quad \text { (discrete derivative), } \\
D[u] & =\sum_{y \in Y} r(y)(d u(y))^{2} \quad \text { (Dirichlet sum), }
\end{aligned}
$$

2000 Mathematics Subject Classification : Primary 31C20; Secondary 31C25. 


$$
\begin{aligned}
D[u, v] & \left.=\sum_{y \in Y} r(y) d u(y) d v(y) \quad \text { (Dirichlet mutual sum }\right) \\
\Delta u(x) & =\sum_{y \in Y} K(x, y) d u(y) \quad \text { (Laplacian), } \\
\Delta^{2} u(x) & =\Delta(\Delta u)(x) \quad(\text { bi-Laplacian }), \\
\mathbf{D}(N) & =\{u \in L(X): D[u]<\infty\} \quad \text { (Dirichlet space) } \\
\mathbf{B D}(N) & =\{u \in \mathbf{D}(N): u \text { is bounded }\} \\
\mathbf{H}(N) & =\{u \in L(X): \Delta u(x)=0 \text { on } X\} \\
\mathbf{H D}(N) & =\mathbf{H}(N) \cap \mathbf{D}(N), \\
\mathbf{D}^{(2)}(N) & =\{u \in L(X): D[\Delta u]<\infty\} \\
\mathbf{H}^{(2)}(N) & =\{u \in L(X): \Delta u \in \mathbf{H}(N)\} \\
\mathbf{Q} \mathbf{P}(N) & =\{u \in L(X): \Delta u=-1, u \geq 0\} \\
\mathbf{H}^{(2)} \mathbf{D}(N) & =\mathbf{H}^{(2)}(N) \cap \mathbf{D}(N) \\
\mathbf{H}^{(2)} \mathbf{D}^{(2)}(N) & =\mathbf{H}^{(2)}(N) \cap \mathbf{D}^{(\mathbf{2})}(N)
\end{aligned}
$$

It is well-known that $\mathbf{D}(N)$ is a Hilbert space with respect to the norm

$$
\|u\|_{2}=\left(D[u]+u\left(x_{0}\right)^{2}\right)^{1 / 2}
$$

for a fixed node $x_{0} \in X$ (cf. [4, Proposition 2.1], [2, Section 9.3]). Denote by $\mathbf{D}_{0}(N)$ the closure of $L_{0}(X)$ in the space $\mathbf{D}(N)$. We say that $N$ is of hyperbolic type if $\mathbf{D}(N) \neq \mathbf{D}_{0}(N)$. In case $N$ is of hyperbolic type, for every $a \in X$ there exists a unique function $u \in L(X)$ which satisfies

$$
u \in \mathbf{D}_{0}(N) \text { and } \Delta u(x)=-\varepsilon_{a}(x),
$$

where $\varepsilon_{a}(x)=0$ if $x \neq a$ and $\varepsilon_{a}(a)=1$. Denote this function by $g_{a}(x)$ and call the Green function of $N$ with pole at $a$.

For $w \in L(Y)$, define $\partial w \in X$ and $H[w]$ by

$$
\partial w(x)=\sum_{y \in Y} K(x, y) w(y)
$$




$$
H[w]=\sum_{y \in Y} r(y) w(y)^{2}
$$

We introduce the following three conditions: We say that the network $N$ satisfies condition (LD) if there exists $0<c<\infty$ such that

$$
D[\Delta f] \leq c D[f]
$$

for all $f \in L_{0}(X)$ (cf. [1]). In case network is of hyperbolic type, a function $u \in L(X)$ is said to satisfy property $(\mathrm{P})$ if

$$
\sum_{x \in X} g_{a}(x)(\Delta u(x))^{2}<\infty
$$

and to satisfy property (SP) if

$$
\sum_{x \in X} g_{a}(x)|\Delta u(x)|<\infty
$$

where $g_{a}$ denotes the Green function of $N$ with pole at $a$. Properties $(\mathrm{P})$ and (SP) were introduced in [3]. In Section 2 we study some sufficient conditions for (LD), in Section 4 some conditions for $(\mathrm{P})$ and in Section 5 some sufficient conditions for (SP). As an application, in Section 5 we prove a Riesz Decomposition theorem for functions in $\mathbf{D}(N)$ satisfying various conditions.

\section{Relations between $D[u]$ and $D[\Delta u]$}

As in [1], we define the arc-arc incidence function $b\left(y, y^{\prime}\right)$ by

$$
b\left(y, y^{\prime}\right)=\sum_{x \in X} K(x, y) K\left(x, y^{\prime}\right)
$$

For $w \in L(Y)$, we define $B_{r} w \in L(Y)$ by

$$
B_{r} w(y)=r(y)^{-1} \sum_{y^{\prime} \in Y} b\left(y, y^{\prime}\right) w\left(y^{\prime}\right)=r(y)^{-1} \sum_{y^{\prime} \in Y(e(y))} b\left(y, y^{\prime}\right) w\left(y^{\prime}\right)
$$

where we mean $Y(e(y))=\bigcup_{x \in e(y)} Y(x)$. For $u \in L(X)$ and $f \in L_{0}(X)$, the following relations will be used frequently: 


$$
\begin{gathered}
\Delta u=\partial d u, \quad d \Delta u=-B_{r} d u, \quad D[\Delta u]=H\left[B_{r} d u\right], \\
D[u, f]=-\sum_{x \in X} u(x) \Delta f(x)=-\sum_{x \in X} f(x) \Delta u(x) .
\end{gathered}
$$

Taking $u=1$ in the formula for $D[u, f]$, we see for $f \in L_{0}(X)$

$$
\sum_{x \in X} \Delta f(x)=0
$$

We show some sufficient conditions of condition (LD).

Lemma 2.1 Denote by $\gamma(y)$ the number of arcs in $Y(e(y))$. For $u \in L(X)$, the following inequality holds:

$$
D[\Delta u] \leq 4 \sum_{y \in Y} r(y)^{-1} \gamma(y) \sum_{y^{\prime} \in Y(e(y))} d u\left(y^{\prime}\right)^{2} .
$$

Proof. Since $\left|b\left(y, y^{\prime}\right)\right| \leq 2$, we have

$$
\left|B_{r} w(y)\right| \leq 2 r(y)^{-1} \sum_{y^{\prime} \in Y(e(y))}\left|w\left(y^{\prime}\right)\right|
$$

so that by the Cauchy-Schwarz Inequality

$$
\left|B_{r} w(y)\right|^{2} \leq 4 r(y)^{-2}\left(\sum_{y^{\prime} \in Y(e(y))} w\left(y^{\prime}\right)\right)^{2} \leq 4 r(y)^{-2} \gamma(y) \sum_{y^{\prime} \in Y(e(y))} w\left(y^{\prime}\right)^{2} .
$$

Letting $w=d u$ we have

$$
\begin{aligned}
D[\Delta u]=H\left[B_{r} w\right] & =\sum_{y \in Y} r(y)\left|B_{r} w(y)\right|^{2} \\
& \leq 4 \sum_{y \in Y} r(y)^{-1} \gamma(y) \sum_{y^{\prime} \in Y(e(y))} w\left(y^{\prime}\right)^{2} .
\end{aligned}
$$

Theorem 2.1 Assume that there exist $r_{0}>0$ and $\gamma_{0}<\infty$ such that $r(y) \geq r_{0}$ and $\gamma(y) \leq \gamma_{0}$ for all $y \in Y$. For $u \in L(X)$ the following inequality holds: 


$$
D[\Delta u] \leq \frac{4 \gamma_{0}^{2}}{r_{0}} \sum_{y \in Y} d u(y)^{2} \leq \frac{4 \gamma_{0}^{2}}{r_{0}^{2}} D[u]
$$

Especially $N$ satisfies condition (LD).

Proof. Lemma 2.1 shows that

$$
\begin{aligned}
D[\Delta u] & \leq 4 \sum_{y \in Y} r(y)^{-1} \gamma(y) \sum_{y^{\prime} \in Y(e(y))} d u\left(y^{\prime}\right)^{2} \leq \frac{4 \gamma_{0}}{r_{0}} \sum_{y \in Y} \sum_{y^{\prime} \in Y(e(y))} d u\left(y^{\prime}\right)^{2} \\
& \leq \frac{4 \gamma_{0}^{2}}{r_{0}} \sum_{y \in Y} d u(y)^{2} \leq \frac{4 \gamma_{0}^{2}}{r_{0}^{2}} \sum_{y \in Y} r(y) d u(y)^{2}=\frac{4 \gamma_{0}^{2}}{r_{0}^{2}} D[u]
\end{aligned}
$$

as required.

Corollary 2.1 Assume that $\gamma(y) \leq \gamma_{0}<\infty$ and $r(y) \equiv 1$ on $Y$. Then $D[\Delta u] \leq 4 \gamma_{0}^{2} D[u]$ for every $u \in L(X)$.

Remark 2.1 Denote by $\nu(x)$ the number of arcs which meet $x$, i.e., $\nu(x)=$ $\# Y(x)=\sum_{y \in Y}|K(x, y)|$. Then $\gamma(y) \leq \nu(a)+\nu(b)$, where $e(y)=\{a, b\}$. If $\nu(x) \leq \nu_{0}$ for $x \in X$, then $\gamma_{0} \leq 2 \nu_{0}$. Corollary 2.1 implies that $D[\Delta u] \leq$ $16 \nu_{0}^{2} D[u]$.

We proved in [1, Proposition 6.1]

Proposition 2.1 Assume that $\nu(x) \leq \nu_{0}$ for all $x \in X$ and $r(y) \equiv 1$ on $Y$. Then $D[\Delta u] \leq 8 \nu_{0}^{2} D[u]$ for every $u \in L(X)$.

Theorem 2.2 Let $\delta$ be the number defined by

$$
\delta=\sum_{y \in Y} \sum_{y^{\prime} \in Y} r(y)^{-1}\left|b\left(y, y^{\prime}\right)\right|^{2} r\left(y^{\prime}\right)^{-1} .
$$

Then $D[\Delta u] \leq \delta D[u]$. Especially $N$ satisfies condition (LD) if $\delta<\infty$.

Proof. By Cauchy-Schwarz's inequality, we have

$$
\begin{aligned}
\left(B_{r} w(y)\right)^{2} & \leq r(y)^{-2}\left(\sum_{y^{\prime} \in Y}\left|b\left(y, y^{\prime}\right)\right|^{2} r\left(y^{\prime}\right)^{-1}\right)\left(\sum_{y^{\prime} \in Y} r\left(y^{\prime}\right) w\left(y^{\prime}\right)^{2}\right) \\
& =r(y)^{-2}\left(\sum_{y^{\prime} \in Y}\left|b\left(y, y^{\prime}\right)\right|^{2} r\left(y^{\prime}\right)^{-1}\right) H[w] .
\end{aligned}
$$


Letting $w=d u$ we have

$$
\begin{aligned}
D[\Delta u] & =H\left[B_{r} w\right]=\sum_{y \in Y} r(y)\left(B_{r} w(y)\right)^{2} \\
& \leq\left(\sum_{y \in Y} \sum_{y^{\prime} \in Y} r(y)^{-1}\left|b\left(y, y^{\prime}\right)\right|^{2} r\left(y^{\prime}\right)^{-1}\right) H[w]=\delta D[u] .
\end{aligned}
$$

Corollary 2.2 $D[\Delta u] \leq 4\left(\sum_{y \in Y} r(y)^{-1}\right)^{2} D[u]$.

Proof. It suffices to note that $\left|b\left(y, y^{\prime}\right)\right| \leq 2$.

Remark 2.2 There is no relation between $D[\Delta u]$ and $D[u]$ in general. We show this by using the following network:

Proposition 2.2 Let $X=\left\{x_{n}\right\}_{n \geq 0}$ and $Y=\left\{y_{n}\right\}_{n \geq 1}$. Let $K\left(x_{n}, y_{n}\right)=1$, $K\left(x_{n-1}, y_{n}\right)=-1$ for $n \geq 1$ and $K(x, y)=0$ for any other pairs. We call this network a linear network. Assume that $\inf _{n} r\left(y_{n}\right)=0$. Then condition (LD) does not hold for $\langle X, Y, K, r\rangle$.

Proof. Let

$$
u_{n}\left(x_{k}\right)= \begin{cases}1 & \text { if } k<n \\ 0 & \text { if } k \geq n\end{cases}
$$

Then

$$
\begin{aligned}
d u_{n}\left(y_{n}\right) & =-r\left(y_{n}\right)^{-1}\left(u_{n}\left(x_{n}\right)-u_{n}\left(x_{n-1}\right)\right)=r\left(y_{n}\right)^{-1}, \\
d u_{n}\left(y_{k}\right) & =-r\left(y_{k}\right)^{-1}\left(u_{n}\left(x_{k}\right)-u_{n}\left(x_{k-1}\right)\right)=0 \quad \text { for } k \neq n ; \\
\Delta u_{n}\left(x_{n-1}\right) & =d u_{n}\left(y_{n-1}\right)-d u_{n}\left(y_{n}\right)=-r\left(y_{n}\right)^{-1}, \\
\Delta u_{n}\left(x_{n}\right) & =d u_{n}\left(y_{n}\right)-d u_{n}\left(y_{n+1}\right)=r\left(y_{n}\right)^{-1} ; \\
d \Delta u_{n}\left(y_{n}\right) & =-r\left(y_{n}\right)^{-1}\left(\Delta u_{n}\left(x_{n}\right)-\Delta u_{n}\left(x_{n-1}\right)\right)=-2 r\left(y_{n}\right)^{-2} .
\end{aligned}
$$

Therefore

$$
\begin{gathered}
D\left[u_{n}\right]=r\left(y_{n}\right) \times\left(r\left(y_{n}\right)^{-1}\right)^{2}=r\left(y_{n}\right)^{-1}, \\
D\left[\Delta u_{n}\right] \geq r\left(y_{n}\right) \times\left(-2 r\left(y_{n}\right)^{-2}\right)^{2}=4 r\left(y_{n}\right)^{-3} .
\end{gathered}
$$


We have

$$
\inf _{n} \frac{D\left[u_{n}\right]}{D\left[\Delta u_{n}\right]} \leq \inf _{n} \frac{r\left(y_{n}\right)^{2}}{4}=0
$$

as required.

Corollary 2.3 A linear network satisfies condition (LD) if and only if $\inf _{n} r\left(y_{n}\right)>0$.

Proof. Theorem 2.1 and Proposition 2.2 show the assertion.

A network with $\inf _{y \in Y} r(y)>0$ does not satisfy condition (LD) in general.

Example 2.1 Let $X=\left\{x_{n}\right\}_{n \geq 0} \cup\left\{z_{n, j}\right\}_{n \geq 1,1 \leq j \leq n}$ and $Y=$ $\left\{y_{n, j}^{+}, y_{n, j}^{-}\right\}_{n \geq 1,1 \leq j \leq n} . \quad$ Let $K\left(x_{n}, y_{n, j}^{+}\right)=1, K\left(z_{n, j}, y_{n, j}^{+}\right)=-1$, $K\left(x_{n-1}, y_{n, j}^{-}\right)=-1, K\left(z_{n, j}, y_{n, j}^{-}\right)=1$ for $n \geq 1$ and $1 \leq j \leq n$ and $K(x, y)=0$ for any other pairs. Let $r\left(y_{n, j}^{+}\right) \equiv r\left(y_{n, j}^{-}\right) \equiv 1$. Let

$$
u_{n}\left(x_{k}\right)=\left\{\begin{array}{ll}
2 & \text { if } k<n ; \\
0 & \text { if } k \geq n,
\end{array} \quad u_{n}\left(z_{k, j}\right)= \begin{cases}2 & \text { if } k<n \\
1 & \text { if } k=n \\
0 & \text { if } k>n\end{cases}\right.
$$

Then

$$
\begin{aligned}
& d u_{n}\left(y_{k, j}^{-}\right)=-\left(u_{n}\left(z_{k, j}\right)-u_{n}\left(x_{k-1}\right)\right)= \begin{cases}1 & \text { if } k=n \\
0 & \text { if } k \neq n\end{cases} \\
& d u_{n}\left(y_{k, j}^{+}\right)=-\left(u_{n}\left(x_{k}\right)-u_{n}\left(z_{k, j}\right)\right)= \begin{cases}1 & \text { if } k=n \\
0 & \text { if } k \neq n\end{cases} \\
& \Delta u_{n}\left(x_{k}\right)=\sum_{j=1}^{k} d u_{n}\left(y_{k, j}^{+}\right)-\sum_{j=1}^{k+1} d u_{n}\left(y_{k+1, j}^{-}\right)= \begin{cases}-n & \text { if } k=n-1 ; \\
n & \text { if } k=n ; \\
0 & \text { otherwise, }\end{cases} \\
& \Delta u_{n}\left(z_{k, j}\right)=d u_{n}\left(y_{k, j}^{-}\right)-d u_{n}\left(y_{k, j}^{+}\right)=0,
\end{aligned}
$$




$$
\begin{aligned}
& d \Delta u_{n}\left(y_{k, j}^{-}\right)=-\left(\Delta u_{n}\left(z_{k, j}\right)-\Delta u_{n}\left(x_{k-1}\right)\right)= \begin{cases}-n & \text { if } k=n \\
n & \text { if } k=n+1 \\
0 & \text { otherwise }\end{cases} \\
& d \Delta u_{n}\left(y_{k, j}^{+}\right)=-\left(\Delta u_{n}\left(x_{k}\right)-\Delta u_{n}\left(z_{k, j}\right)\right)= \begin{cases}n & \text { if } k=n-1 \\
-n & \text { if } k=n \\
0 & \text { otherwise }\end{cases}
\end{aligned}
$$

and

$$
\begin{aligned}
D\left[u_{n}\right]= & \sum_{k=1}^{\infty} \sum_{j=1}^{k}\left(d u_{n}\left(y_{k, j}^{-}\right)^{2}+d u_{n}\left(y_{k, j}^{+}\right)^{2}\right)=\sum_{j=1}^{n}\left(1^{2}+1^{2}\right)=2 n, \\
D\left[\Delta u_{n}\right]= & \sum_{k=1}^{\infty} \sum_{j=1}^{k}\left(d \Delta u_{n}\left(y_{k, j}^{-}\right)^{2}+d \Delta u_{n}\left(y_{k, j}^{+}\right)^{2}\right) \\
= & \sum_{j=1}^{n-1} d \Delta u_{n}\left(y_{n-1, j}^{+}\right)^{2}+\sum_{j=1}^{n}\left(d \Delta u_{n}\left(y_{n, j}^{-}\right)^{2}+d \Delta u_{n}\left(y_{n, j}^{+}\right)^{2}\right) \\
& +\sum_{j=1}^{n+1} d \Delta u_{n}\left(y_{n+1, j}^{-}\right)^{2} \\
= & (n-1) n^{2}+n\left((-n)^{2}+(-n)^{2}\right)+(n+1) n^{2}=4 n^{3} .
\end{aligned}
$$

We have

$$
\frac{D\left[u_{n}\right]}{D\left[\Delta u_{n}\right]}=\frac{2 n}{4 n^{3}} \rightarrow 0
$$

as $n \rightarrow \infty$.

\section{Condition (LD)}

We proved in [1, Lemma 6.1$]$

Lemma 3.1 Condition (LD) implies $\Delta\left(\mathbf{D}_{0}(N)\right) \subset \mathbf{D}_{0}(N)$.

Theorem 3.1 Assume condition (LD). Then $\mathbf{D}(N) \subset \mathbf{D}^{(2)}(N)$. 
Proof. Let $u \in \mathbf{D}(N)$. There exist $v \in \mathbf{D}_{0}(N)$ and $h \in \mathbf{H D}(N)$ such that $u=v+h$ by Royden's decomposition (cf. [5, Theorem 4.1], [2, Exercise 9.6(f)]). Since $\Delta u=\Delta v+\Delta h=\Delta v$, we have $D[\Delta u]=D[\Delta v]<\infty$ by Lemma 3.1.

Lemma 3.2 Assume condition (LD). Then there exists $c>0$ such that $D[\Delta u] \leq c D[u]$ for all $u \in \mathbf{D}(N)$.

Proof. By Royden's decomposition we find $v \in \mathbf{D}_{0}(N)$ and $h \in \mathbf{H D}(N)$ such that $u=v+h$. Let $\left\{f_{n}\right\}_{n}$ be a sequence in $L_{0}(X)$ such that $\left\|v-f_{n}\right\|_{2} \rightarrow$ 0 as $n \rightarrow \infty$. We have $D\left[f_{n}, h\right]=-\sum_{x \in X} f_{n}(x) \Delta h(x)=0$ and

$$
\left|D[v, h]-D\left[f_{n}, h\right]\right|=\left|D\left[v-f_{n}, h\right]\right| \leq D\left[v-f_{n}\right]^{1 / 2} D[h]^{1 / 2} \rightarrow 0
$$

as $n \rightarrow \infty$. These imply $D[v, h]=0$, and that

$$
D[u]=D[v]+D[v, h]+D[h]=D[v]+D[h] \geq D[v] .
$$

Since $\left\{f_{n}(x)\right\}_{n}$ converges pointwise to $v(x)$, we see that $\left\{\Delta f_{n}(x)\right\}_{n}$ converges pointwise to $\Delta v(x)$, so that by Fatou's lemma

$$
D[\Delta v] \leq \liminf _{n \rightarrow \infty} D\left[\Delta f_{n}\right] .
$$

Condition (LD) implies that $D\left[\Delta f_{n}\right] \leq c D\left[f_{n}\right]$ for all $n$. We have

$$
D[\Delta u]=D[\Delta v] \leq \liminf _{n \rightarrow \infty} D\left[\Delta f_{n}\right] \leq c \liminf _{n \rightarrow \infty} D\left[f_{n}\right]=c D[v] \leq c D[u]
$$

as required.

Proposition 3.1 Assume condition (LD). Then $D[h, \Delta v]=0$ for $h \in$ $\mathbf{H}^{(2)} \mathbf{D}(N)$ and $v \in \mathbf{D}_{0}(N)$.

Proof. Let $\left\{f_{n}\right\}_{n}$ be a sequence in $L_{0}(X)$ such that $\left\|v-f_{n}\right\|_{2} \rightarrow 0$ as $n \rightarrow \infty$. Since $\Delta f_{n} \in L_{0}(X)$, we have

$$
\begin{aligned}
& D\left[h, \Delta f_{n}\right]=-\sum_{x \in X}(\Delta h(x))\left(\Delta f_{n}(x)\right) \\
& =D\left[\Delta h, f_{n}\right]=-\sum_{x \in X}\left(\Delta^{2} h(x)\right) f_{n}(x)=0 .
\end{aligned}
$$


Lemma 3.2 shows that

$$
\begin{aligned}
D[h, \Delta v] & =D\left[h, \Delta v-\Delta f_{n}\right] \leq D[h]^{1 / 2} D\left[\Delta\left(v-f_{n}\right)\right]^{1 / 2} \\
& \leq c^{1 / 2} D[h]^{1 / 2} D\left[v-f_{n}\right]^{1 / 2} \rightarrow 0
\end{aligned}
$$

as $n \rightarrow \infty$, so that $D[h, \Delta v]=0$.

Proposition 3.2 Let $h \in \mathbf{H}^{(2)} \mathbf{D}^{(2)}(N)=\mathbf{H}^{(2)}(N) \cap \mathbf{D}^{(2)}(N)$. If condition (LD) is fulfilled, then $D[\Delta h, \Delta v]=0$ for every $v \in \mathbf{D}_{0}(N)$.

Proof. We find $\left\{f_{n}\right\}_{n} \subset L_{0}(X)$ such that $\left\|v-f_{n}\right\|_{2} \rightarrow 0$. Then $D\left[\Delta h, \Delta f_{n}\right]=D\left[\Delta^{2} h, f_{n}\right]=0$. Using Lemma 3.1 we know that $D[\Delta h, \Delta v]$ is well-defined and, using Lemma 3.2, that

$$
D[\Delta h, \Delta v] \leq D[\Delta h]^{1 / 2} D\left[\Delta\left(v-f_{n}\right)\right]^{1 / 2} \leq c^{1 / 2} D[\Delta h]^{1 / 2} D\left[v-f_{n}\right]^{1 / 2} \rightarrow 0
$$

as $n \rightarrow \infty$.

Proposition 3.3 Assume that $N$ is of hyperbolic type and that condition (LD) is fulfilled. Then $\mathbf{D}_{0}(N) \cap \mathbf{H}^{(2)}(N)=\{0\}$.

Proof. Let $u \in \mathbf{D}_{0}(N)$ and $\Delta^{2} u=0$. Then $\Delta u \in \mathbf{D}_{0}(N)$ by Lemma 3.1, so that $\Delta u \in \mathbf{H D}(N)$. Since $N$ is of hyperbolic type, we have $\mathbf{D}_{0}(N) \cap$ $\mathbf{H D}(N)=\{0\}\left(\right.$ see $\left[5\right.$, Lemma 1.3]), so that $\Delta u=0$. Namely $u \in \mathbf{D}_{0}(N) \cap$ $\operatorname{HD}(N)$, and hence $u=0$.

Corollary 3.1 Assume that $N$ is of hyperbolic type and that condition (LD) is fulfilled. Then $\mathbf{H}^{(2)} \mathbf{D}(N)=\mathbf{H D}(N)$.

Proof. Clearly, $\mathbf{H D}(N) \subset \mathbf{H}^{(2)} \mathbf{D}(N)$. Let $u \in \mathbf{H}^{(2)} \mathbf{D}(N)$. There exist $h \in \mathbf{H D}(N)$ and $v \in \mathbf{D}_{0}(N)$ such that $u=h+v$. Then $\Delta^{2} v=\Delta^{2} u=0$. Proposition 3.3 shows that $v=0$, and hence $u=h \in \mathbf{H D}(N)$.

Let $\left\{N_{n}=\left\langle X_{n}, Y_{n}, K_{n}, r_{n}\right\rangle\right\}_{n}$ be an exhaustion of $N$ and $a \in X$. The Green function $g_{a}^{(n)}$ of $N_{n}$ with pole at $a$ is defined by

$$
\Delta g_{a}^{(n)}(x)=-\varepsilon_{a}(x)\left(x \in X_{n}\right), \quad g_{a}^{(n)}(x)=0\left(x \in X \backslash X_{n}\right)
$$

It is well-known that $g_{a}^{(n)}(x) \leq g_{a}^{(n+1)}(x)$ on $X$ and $g_{a}^{(n)}(x) \rightarrow g_{a}(x)$ as $n \rightarrow \infty$ for each $x \in X$ (cf. [5, Remark 3.1]). Noting $D\left[g_{a}\right]=g_{a}(a)$ and 


$$
D\left[g_{a}^{(n)}\right]=-\sum_{x \in X} g_{a}^{(n)}(x), \quad D\left[g_{a}^{(n)}-g_{a}\right]=g_{a}(a)-g_{a}^{(n)}(a)
$$

we have

Lemma 3.3 $\lim _{n \rightarrow \infty} D\left[g_{a}^{(n)}\right]=D\left[g_{a}\right]$ and $\lim _{n \rightarrow \infty} D\left[g_{a}^{(n)}-g_{a}\right]=0$

Theorem 3.2 Assume that $N$ is of hyperbolic type and satisfies condition (LD). If $u \in \mathbf{D}(N)$, then $\sum_{x \in X}(\Delta u(x))^{2}<\infty$.

Proof. Let $\left\{N_{n}=\left\langle X_{n}, Y_{n}, K_{n}, r_{n}\right\rangle\right\}_{n}$ be an exhaustion of $N$ and let $g_{z}^{(n)}$ be the Green function of $N_{n}$ with pole at $z \in X_{n}$. We set $g_{z}^{(n)}(x)=0$ for $x \in X \backslash X_{n}$ and put

$$
f_{n}(x)=\sum_{z \in X_{n}} g_{z}^{(n)}(x) \Delta u(z)
$$

Then

$$
f_{n}(x)=0 \quad \text { on } X \backslash X_{n} \text { and } \quad \Delta f_{n}(x)=-\Delta u(x) \text { on } X_{n} .
$$

Especially $\left\{\Delta f_{n}(x)\right\}_{n}$ converges pointwise to $-\Delta u(x)$. By our assumption, there exists a constant $c>0$ such that $D\left[\Delta f_{n}\right] \leq c D\left[f_{n}\right]$ for all $n$.

Let $h_{n}=u+f_{n}$. Then $h_{n}$ is harmonic on $X_{n}$ and

$$
\begin{aligned}
D[u] & =D\left[h_{n}-f_{n}\right]=D\left[f_{n}\right]+D\left[h_{n}\right]-2 D\left[f_{n}, h_{n}\right] \\
& =D\left[f_{n}\right]+D\left[h_{n}\right]+2 \sum_{x \in X_{n}} f_{n}(x) \Delta h_{n}(x)=D\left[f_{n}\right]+D\left[h_{n}\right],
\end{aligned}
$$

so that $D\left[f_{n}\right] \leq D[u]$. We have

$$
\begin{aligned}
\sum_{x \in X}\left(\Delta f_{n}(x)\right)^{2} & =-D\left[f_{n}, \Delta f_{n}\right] \leq D\left[f_{n}\right]^{1 / 2} D\left[\Delta f_{n}\right]^{1 / 2} \\
& \leq c^{1 / 2} D\left[f_{n}\right] \leq c^{1 / 2} D[u]
\end{aligned}
$$

By Fatou's Lemma, we have

$$
\sum_{x \in X}(\Delta u(x))^{2} \leq \liminf _{n \rightarrow \infty} \sum_{x \in X}\left(\Delta f_{n}(x)\right)^{2} \leq c^{1 / 2} D[u]<\infty .
$$




\section{The metric growth of the Laplacian}

The metric growth of the Laplacian of $u$ is said to be so slow in [3] if $u$ satisfies property $(\mathrm{P})$. As a discrete analog of [3, Theorem VII.1.1], we have

Theorem 4.1 Let $N$ be of hyperbolic type and $u \in \mathbf{D}(N)$. If $\Delta u \in$ $\mathbf{B D}(N)$, then $u$ satisfies property $(\mathrm{P})$.

Proof. Let $a \in X$ and $\left\{N_{n}=\left\langle X_{n}, Y_{n}, K_{n}, r_{n}\right\rangle\right\}_{n}$ be an exhaustion of $N$ such that $a \in X_{1}$ and let $g_{a}^{(n)}$ be the Green function of $N_{n}$ with pole at $a$. We set $g_{a}^{(n)}(x)=0$ for $x \in X \backslash X_{n}$. Then $g_{a}^{(n)} \in L_{0}(X)$ and $D\left[g_{a}^{(n)}-g_{a}\right] \rightarrow 0$ as $n \rightarrow \infty$. We put

$$
\varphi_{n}(x)=-g_{a}^{(n)}(x) \Delta u(x)
$$

By our assumption, there exists $c_{1}>0$ such that $|\Delta u(x)| \leq c_{1}$ on $X$. We shall show that

$$
\left|d \varphi_{n}(y)\right| \leq M\left(\left|d g_{a}^{(n)}(y)\right|+|d \Delta u(y)|\right)
$$

where $M=\max \left(c_{1}, g_{a}(a)\right)$. Let $e(y)=\left\{x_{1}, x_{2}\right\}$. Since $g_{a}^{(n)}(x) \leq g_{a}^{(n)}(a) \leq$ $g_{a}(a)$

$$
\begin{aligned}
\left|r(y) d \varphi_{n}(y)\right| & =\left|g_{a}^{(n)}\left(x_{1}\right) \Delta u\left(x_{1}\right)-g_{a}^{(n)}\left(x_{2}\right) \Delta u\left(x_{2}\right)\right| \\
& \leq\left|g_{a}^{(n)}\left(x_{1}\right)\right|\left|\Delta u\left(x_{1}\right)-\Delta u\left(x_{2}\right)\right|+\left|g_{a}^{(n)}\left(x_{1}\right)-g_{a}^{(n)}\left(x_{2}\right)\right|\left|\Delta u\left(x_{2}\right)\right| \\
& \leq g_{a}(a)|r(y) d \Delta u(y)|+c_{1}\left|r(y) d g_{a}^{(n)}(y)\right| \\
& \leq M r(y)\left(\left|d g_{a}^{(n)}(y)\right|+|d \Delta u(y)|\right) .
\end{aligned}
$$

We have

$$
\begin{aligned}
D\left[\varphi_{n}\right] & \leq \sum_{y \in Y} r(y)\left(M\left(\left|d g_{a}^{(n)}(y)\right|+|d \Delta u(y)|\right)\right)^{2} \\
& \leq 2 M^{2}\left(D\left[g_{a}^{(n)}\right]+D[\Delta u]\right) \rightarrow 2 M^{2}\left(D\left[g_{a}\right]+D[\Delta u]\right)
\end{aligned}
$$

There is a constant $c_{2}>0$ such that $D\left[\varphi_{n}\right] \leq c_{2}$. Since $\varphi_{n} \in L_{0}(X)$, we have 


$$
\begin{aligned}
\sum_{x \in X} g_{a}^{(n)}(x)(\Delta u(x))^{2} & =-\sum_{x \in X} \varphi_{n}(x) \Delta u(x)=D\left[\varphi_{n}, u\right] \\
& \leq D\left[\varphi_{n}\right]^{1 / 2} D[u]^{1 / 2} \leq c_{2}^{1 / 2} D[u]^{1 / 2}
\end{aligned}
$$

By Fatou's lemma

$$
\sum_{x \in X} g_{a}(x)(\Delta u(x))^{2} \leq \liminf _{n \rightarrow \infty} \sum_{x \in X} g_{a}^{(n)}(x)(\Delta u(x))^{2} \leq c_{2}^{1 / 2} D[u]^{1 / 2},
$$

that is, $u$ satisfies property $(\mathrm{P})$.

We prepare

Lemma 4.1 The following relation holds for $f_{1}, f_{2} \in L(X)$ :

$$
\Delta\left(f_{1} f_{2}\right)(x)=\left(\Delta f_{1}(x)\right) f_{2}(x)+f_{1}(x)\left(\Delta f_{2}(x)\right)+\sum_{y \in Y(x)} r(y) d f_{1}(y) d f_{2}(y)
$$

Proof. For $x \in X$ and $y \in Y(x)$ we let $\mathrm{n}_{x}(y)$ be a unique node with

$$
K(x, y) K\left(\mathrm{n}_{x}(y), y\right)=-1 .
$$

Then

$$
\begin{aligned}
d u(y) & =-r(y)^{-1}\left(K(x, y) u(x)+K\left(\mathrm{n}_{x}(y), y\right) u\left(\mathrm{n}_{x}(y)\right)\right) \\
& =-r(y)^{-1} K(x, y)\left(u(x)-u\left(\mathrm{n}_{x}(y)\right)\right), \\
\Delta u(x) & =\sum_{y \in Y(x)} K(x, y) d u(y)=-\sum_{y \in Y(x)} r(y)^{-1}\left(u(x)-u\left(\mathrm{n}_{x}(y)\right)\right) .
\end{aligned}
$$

We have

$$
\begin{aligned}
& \Delta\left(f_{1} f_{2}\right)(x)-\left(\Delta f_{1}(x)\right) f_{2}(x)-f_{1}(x)\left(\Delta f_{2}(x)\right) \\
& =-\sum_{y \in Y(x)} r(y)^{-1}\left(f_{1}(x) f_{2}(x)-f_{1}\left(\mathrm{n}_{x}(y)\right) f_{2}\left(\mathrm{n}_{x}(y)\right)\right) \\
& \quad+\sum_{y \in Y(x)} r(y)^{-1}\left(f_{1}(x)-f_{1}\left(\mathrm{n}_{x}(y)\right)\right) f_{2}(x) \\
& \quad+f_{1}(x) \sum_{y \in Y(x)} r(y)^{-1}\left(f_{2}(x)-f_{2}\left(\mathrm{n}_{x}(y)\right)\right)
\end{aligned}
$$




$$
\begin{aligned}
& =\sum_{y \in Y(x)} r(y)^{-1}\left(f_{1}(x) f_{2}(x)+f_{1}\left(\mathrm{n}_{x}(y)\right) f_{2}\left(\mathrm{n}_{x}(y)\right)\right. \\
& \left.\quad-f_{1}\left(\mathrm{n}_{x}(y)\right) f_{2}(x)-f_{1}(x) f_{2}\left(\mathrm{n}_{x}(y)\right)\right) \\
& =\sum_{y \in Y(x)} r(y)^{-1}\left(f_{1}(x)-f_{1}\left(\mathrm{n}_{x}(y)\right)\right)\left(f_{2}(x)-f_{2}\left(\mathrm{n}_{x}(y)\right)\right),
\end{aligned}
$$

using (1),

$$
\begin{aligned}
& =\sum_{y \in Y(x)} r(y)^{-1}\left(-r(y) K(x, y) d f_{1}(y)\right)\left(-r(y) K(x, y) d f_{2}(y)\right) \\
& =\sum_{y \in Y(x)} r(y) d f_{1}(y) d f_{2}(y) .
\end{aligned}
$$

This completes the proof.

As a discrete analog of [3, Theorem VII.1.2], we shall prove

Theorem 4.2 Assume that $N$ is of hyperbolic type. If $u \in \mathbf{H}^{(2)} \mathbf{D}^{(2)}(N) \cap$ $\mathbf{B D}(N)$, then $u$ satisfies property $(\mathrm{P})$.

Proof. Let $\left\{N_{n}=\left\langle X_{n}, Y_{n}, K_{n}, r_{n}\right\rangle\right\}_{n}$ be an exhaustion of $N$ and let $g_{z}^{(n)}$ be the Green function of $N_{n}$ with pole at $z \in X_{n}$. We set $g_{z}^{(n)}(x)=0$ for $x \in X \backslash X_{n}$. Now we consider $f_{n} \in L(X)$ defined by

$$
f_{n}(x)=\sum_{z \in X_{n}} g_{z}^{(n)}(x) \Delta u(z)
$$

Then

$$
f_{n}(x)=0 \quad \text { on } X \backslash X_{n} \quad \text { and } \quad \Delta f_{n}(x)=-\Delta u(x) \quad \text { on } X_{n} .
$$

By our assumption, there exists $c>0$ such that $|u(x)| \leq c$ on $X$. We shall show that $\left|f_{n}(x)\right| \leq 2 c$ on $X$. We may assume $x \in X_{n}$. Define $\varphi(z)=g_{z}^{(n)}(x)=g_{x}^{(n)}(z)$ for $z \in X_{n}$ and $\varphi(z)=0$ for $z \in X \backslash X_{n}$. Notice that $\Delta \varphi(z)=-\varepsilon_{x}(z)$ for $z \in X_{n}$. For $z \in \partial X_{n}$ (the outer boundary of $X_{n}$ ), we use (2) and obtain 


$$
\Delta \varphi(z)=-\sum_{y \in Y(z)} r(y)^{-1}\left(\varphi(z)-\varphi\left(\mathrm{n}_{z}(y)\right)\right)=\sum_{\substack{y \in Y(z) ; \\ \mathrm{n}_{z}(y) \in X_{n}}} r(y)^{-1} g_{x}^{(n)}\left(\mathrm{n}_{z}(y)\right) \geq 0 .
$$

Since $\varphi \in L_{0}(X)$, we have

$$
0=\sum_{z \in X} \Delta \varphi(z)=-1+\sum_{z \in \partial X_{n}} \Delta \varphi(z)
$$

and

$$
f_{n}(x)=\sum_{z \in X} \varphi(z) \Delta u(z)=\sum_{z \in X} u(z) \Delta \varphi(z)=-u(x)+\sum_{z \in \partial X_{n}} u(z) \Delta \varphi(z) .
$$

These implies

$$
\left.\left|f_{n}(x)\right| \leq|u(x)|+\sum_{z \in \partial X_{n}} \mid u(z) \Delta \varphi(z)\right] \leq c+c \sum_{z \in \partial X_{n}} \Delta \varphi(z)=2 c .
$$

Applying Lemma 4.1 to $g_{a}^{(n)}(z)$ and $\Delta u(z)$ and using $u \in \mathbf{H}^{(2)}(N)$, we have

$$
\begin{aligned}
\Delta\left(g_{a}^{(n)} \Delta u\right)(z)= & \Delta g_{a}^{(n)}(z) \Delta u(z)+g_{a}^{(n)}(z) \Delta^{2} u(z) \\
& +\sum_{y \in Y(z)} r(y) d g_{a}^{(n)}(y) d \Delta u(y) \\
= & \Delta g_{a}^{(n)}(z) \Delta u(z)+\sum_{y \in Y(z)} r(y) d g_{a}^{(n)}(y) d \Delta u(y) .
\end{aligned}
$$

Thus we have

$$
\begin{aligned}
& \sum_{z \in X} g_{a}^{(n)}(z)(\Delta u(z))^{2}=\sum_{z \in X_{n}} g_{a}^{(n)}(z)(\Delta u(z))^{2} \\
& =-\sum_{z \in X_{n}} g_{a}^{(n)}(z) \Delta f_{n}(z) \Delta u(z)=-\sum_{z \in X} \Delta f_{n}(z) g_{a}^{(n)}(z) \Delta u(z) \\
& =-\sum_{z \in X} f_{n}(z) \Delta\left(g_{a}^{(n)} \Delta u\right)(z)=-\sum_{z \in X_{n}} f_{n}(z) \Delta\left(g_{a}^{(n)} \Delta u\right)(z) \\
& =-\sum_{z \in X_{n}} f_{n}(z) \Delta g_{a}^{(n)}(z) \Delta u(z)-\sum_{z \in X_{n}} \sum_{y \in Y(z)} f_{n}(z) r(y) d g_{a}^{(n)}(y) d \Delta u(y)
\end{aligned}
$$




$$
\begin{aligned}
& =f_{n}(a) \Delta u(a)-\sum_{z \in X_{n}} \sum_{y \in Y(z)} f_{n}(z) r(y) d g_{a}^{(n)}(y) d \Delta u(y) \\
& \leq\left|f_{n}(a) \Delta u(a)\right|+\sum_{z \in X_{n}} \sum_{y \in Y(z)}\left|f_{n}(z) r(y) d g_{a}^{(n)}(y) d \Delta u(y)\right| \\
& \leq 2 c|\Delta u(a)|+4 c \sum_{y \in Y} r(y)\left|d g_{a}^{(n)}(y)\right||d \Delta u(y)| \\
& \leq 2 c|\Delta u(a)|+4 c D\left[g_{a}^{(n)}\right]^{1 / 2} D[\Delta u]^{1 / 2} .
\end{aligned}
$$

Since $D\left[g_{a}^{(n)}\right] \rightarrow D\left[g_{a}\right]$ as $n \rightarrow \infty$, we have

$$
\begin{aligned}
\sum_{z \in X} g_{a}(z)[\Delta u(z)]^{2} & \leq \liminf _{n \rightarrow \infty} \sum_{z \in X} g_{a}^{(n)}(z)(\Delta u(z))^{2} \\
& \leq 2 c|\Delta u(a)|+4 c D\left[g_{a}\right]^{1 / 2} D[\Delta u]^{1 / 2}<\infty
\end{aligned}
$$

This shows that $u$ satisfies property $(\mathrm{P})$.

Theorem 4.3 Assume that $N$ is of hyperbolic type and satisfies condition (LD). Then every $u \in \mathbf{D}(N)$ satisfies property $(\mathrm{P})$.

Proof. $\quad$ Since $g_{a}(x) \leq g_{a}(a)<\infty$, Theorem 3.2 implies

$$
\sum_{x \in X} g_{a}(x)(\Delta u(x))^{2} \leq g_{a}(a) \sum_{x \in X}(\Delta u(x))^{2}<\infty .
$$

\section{Riesz Decomposition}

To study property (SP) introduced in Section 1, we recall the following set of functions on $N$ :

$$
\mathbf{Q P}(N)=\{u \in L(X): \Delta u=-1, u \geq 0\} .
$$

We denote by $\mathbf{O}_{Q P}$ the set of all locally finite infinite networks $N$ such that the set $\mathbf{Q P}(N)$ is the empty set.

We proved in [6, Theorem 3.1]

Lemma 5.1 $N \notin \mathbf{O}_{Q P}$ if and only if $\sum_{x \in X} g_{a}(x)<\infty$ for all $a \in X$.

Notice that if $N \notin \mathbf{O}_{Q P}$, then $N$ is of hyperbolic type, so that there 
exists the Green function $g_{a}(x)$ of $N$ with pole at $a$.

Proposition 5.1 Let $N \notin \mathbf{O}_{Q P}$. Then property (P) implies property (SP) for every $u \in \mathbf{D}(N)$.

Proof. $\quad$ Since $u$ satisfies property (P), we see by Lemma 5.1

$$
\sum_{x \in X} g_{a}(x)|\Delta u(x)| \leq\left(\sum_{x \in X} g_{a}(x)\right)^{1 / 2}\left(\sum_{x \in X} g_{a}(x)(\Delta u(x))^{2}\right)^{1 / 2}<\infty
$$

for every $a \in X$.

We have

Theorem 5.1 Let $N \notin \mathbf{O}_{Q P}$. If $u \in \mathbf{D}(N)$ satisfies property (SP), then $u$ has the following Riesz Decomposition: There exists $h \in \mathbf{H D}(N)$ such that

$$
u(x)=h(x)+\sum_{z \in X} g_{z}(x)(-\Delta u(z))
$$

on $X$.

Proof. By our assumption, the Green potential

$$
v(x):=\sum_{z \in X} g_{z}(x) \Delta u(z) \in L(X)
$$

of a signed measure $\Delta u$ is well-defined. Let $\left\{N_{n}=\left\langle X_{n}, Y_{n}, K_{n}, r_{n}\right\rangle\right\}_{n}$ be an exhaustion of $N$ and let $g_{z}^{(n)}$ be the Green function of $N_{n}$ with pole at $z \in X_{n}$. We set $g_{z}^{(n)}(x)=0$ for $x \in X \backslash X_{n}$ and define $f_{n}$ and $h_{n}$ by

$$
f_{n}(x)=\sum_{z \in X_{n}} g_{z}^{(n)}(x) \Delta u(z), \quad h_{n}=u+f_{n}
$$

Notice that $h_{n}$ is harmonic on $X_{n}$ and

$$
D\left[h_{n}, f_{n}\right]=-\sum_{x \in X}\left(\Delta h_{n}(x)\right) f_{n}(x)=0
$$

so that $D[u]=D\left[h_{n}\right]+D\left[f_{n}\right]$. We see by Lebesgue's dominated convergence theorem that $\left\{f_{n}(x)\right\}_{n}$ converges pointwise to a function $v(x)$ for every 
$x \in X$. Since $\left\{D\left[f_{n}\right]\right\}_{n}$ is bounded, we see that $v \in \mathbf{D}_{0}(N)$ (cf. [7, Theorem 4.1]). Let $h$ be the pointwise limit of $h_{n}$. Then $h=u+v \in \mathbf{D}(N)$ and $h$ is harmonic on $X$, i.e., $h \in \mathbf{H D}(N)$. We have

$$
u(x)=h(x)-v(x)=h(x)+\sum_{z \in X} g_{z}(x)(-\Delta u(z))
$$

for $x \in X$.

Remark 5.1 In the above theorem, we see that $h=0$ if $u \in \mathbf{D}_{0}(N)$.

Corollary 5.1 Let $N \notin \mathbf{O}_{Q P}$. Then $u \in \mathbf{D}(N)$ admits the following Riesz Decomposition:

$$
u(x)=h(x)+\sum_{z \in X} g_{x}(z)(-\Delta u(z)), \quad h \in \mathbf{H D}(N)
$$

if any one of the following conditions is satisfied:

(1) $\Delta u \in \mathbf{B D}(N)$;

(2) $u$ is bounded and $u \in \mathbf{H}^{(2)} \mathbf{D}^{(2)}(N)$;

(3) condition (LD) is satisfied.

Proof. Since $N \notin \mathbf{O}_{Q P}, N$ is of hyperbolic type. One of Theorems 4.1, 4.2 and 4.3 can be applied and implies that $u$ satisfies property $(\mathrm{P})$. Proposition 5.1 shows that $u$ satisfies property (SP). By Theorem 5.1 we have the assertion.

Acknowledgement We would like to thank the referee for his detailed comments which improved significantly the presentation of this paper.

\section{References}

[1 ] Kurata H. and Yamasaki M., Bi-flows on a network. Hokkaido Mathematical Journal, to appear.

[ 2 ] Lyons R. and Peres Y., Probability on trees and networks, http://www.uni-

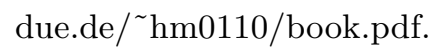

[ 3 ] Sario L., Nakai M., Wang C. and Chung L. O., Classification theory of Riemannian manifolds. Lecture Notes in Mathematics, Vol. 605, SpringerVerlag, Berlin, 1977, Harmonic, quasiharmonic and biharmonic functions.

[ 4 ] Yamasaki M., Parabolic and hyperbolic infinite networks. Hiroshima Math. 
7 (1977), 135-146.

[5] Yamasaki M., Discrete potentials on an infinite network. Mem. Fac. Sci. Shimane Univ. 13 (1979), 31-44.

[6] Yamasaki M., Quasiharmonic classification of infinite networks. Discrete Appl. Math. 2 (1980), 339-344.

[ 7 ] Yamasaki M., Discrete Dirichlet potentials on an infinite network. RIMS Kokyuroku 610 (1987), 51-66.

Hisayasu KuRATA

Yonago National College of Technology

Yonago, Tottori, 683-8502 Japan

E-mail: kurata@yonago-k.ac.jp

Maretsugu YAMASAKI

Matsue, Shimane, 690-0824 Japan

E-mail: yama0565m@mable.ne.jp 